\title{
When crop transgenes wander in California, should we worry?
}

\author{
by Norman C. Ellstrand
}

The movement of transgenes into populations for which they are not intended remains a primary concern for genetically engineered crops. Such gene flow in itself is not a risk. However, we know that the transfer of genes from traditionally improved crops into wild populations has already resulted, on occasion, in the evolution of weeds more difficult to control, as well as an increased extinction risk for rare species. Just like traditional crops, genetically engineered crops could occasionally create the same problems. Currently in California, the movement of transgenes from most commercialized transgenic crops into wild plant populations is unlikely - the exception being canola. However, other transgenic plants have been field-tested in California, and if these become commercialized, in certain cases, transgenes are likely to move into the wild or into other crops of the same species. Such gene flow could result in various problems. The best containment for transgenes may involve risk assessment decisions by scientists embarking on projects to determine whether the proposed combination of organism and trait will pose any problems and if so, to determine how to create a safe product.

\footnotetext{
T 1985 , scientists published the first two papers addressing the potential environmental impacts of genetically engineered crops. California scientists played important roles in writing both. Senior personnel at CalGene, a California-based genetic engineering firm, wrote one paper (Goodman and Newell 1985), and
}
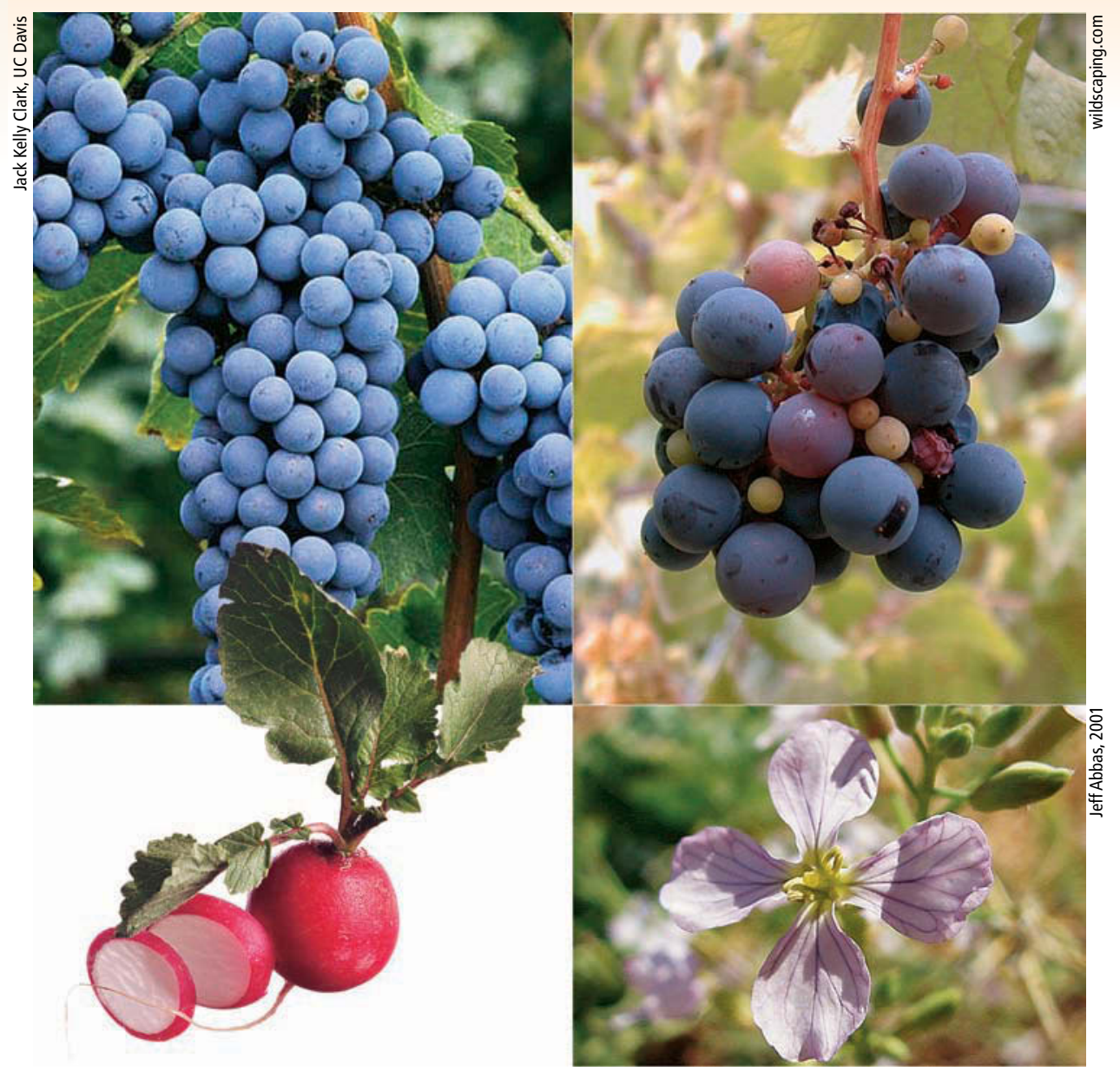

Scientists are studying the implications of gene flow between cultivated crops and their wild relatives, a common phenomenon between plant species. For example, studies have considered, top left, cultivated wine grape (Vitis vinifera) and its wild relative, top right, California wild grape (Vitis californica), as well as, bottom left, crop radish and, bottom right, wild radish (Raphanus sativus).

a UC Berkeley faculty member was senior author of the other (Colwell et al. 1985). Both articles prominently featured the possibility that hybridization could serve as an avenue for the unintended movement of engineered genes (transgenes) from transgenic crops into populations of related weeds. Such movement of genes between species or populations, called "gene flow," in itself does not pose a risk. Gene flow by pollen and seed between cross-compatible populations is not uncommon, and often plays an important role in both evolution and plant breeding (Ellstrand 2003a).

Both papers pointed out that the presence of crop genes in wild populations has long been recognized as a stimulus for the evolution of increased weediness or "superweeds" (Anderson 1949). Goodman and Newell (1985) stated the problem succinctly: "The sexual transfer of genes to weedy species to create a more persistent weed is probably the greatest environmental risk of planting a new variety of crop species."

Other potential environmental problems of transgenic crops were anticipated and discussed in those articles (see sidebar, page 119), but the risks associated with the unintentional movement of engineered genes into populations for which they were not intended continue to receive the most attention in both scientific publications and the popular press. This attention may stem, in part, from the fact that the movement of unwanted crop genes into the environment poses more of a management 
dilemma than unwanted nonliving "pollutants." For example, a single molecule of DDT remains a single molecule or degrades. But a single crop allele occurs within an organism that may have the opportunity to multiply itself - and that allele - repeatedly through reproduction. The fact that unwanted genes can increase their numbers could frustrate attempts at recall or containment. Indeed, almost every general treatment of the environmental impacts of plant biotechnology gives some consideration to gene flow (Dale et al. 2002; Hails 2000; Marvier 2001; NRC 1989, 2000, 2002, 2004; Nickson and Head 1999; Rissler and Mellon 1996; Scientists' Working Group on Biosafety 1998; Snow et al. 2005; Wolfenbarger and Phifer 2000), and a book on the topic was recently published (den Nijs et al. 2004).

Over the last 17 years, my research program has evolved to focus on the topic, addressing the following questions: How likely is it that transgenes will move into and establish in natural populations? And if transgenes do move into wild populations, is there any cause for concern? Traditionally improved crops can serve as models for the behavior of transgenic crops; indeed, the U.S. regulatory framework for transgenic plants is based on this assumption (NRC 1989, 2002). Experience with traditional crops and experiments using them can provide a tremendous amount of information for answering these questions.

\section{Spontaneous hybridization study}

In the early 1990s, the general view was that hybridization between crops and their wild relatives occurred extremely infrequently, even if they were growing in close proximity. This view was probably due to the difficulties breeders sometimes have in creating crop-wild hybrids (Fehr 1987). My research group set out to measure spontaneous hybridization between wild radish (Raphanus sativus), an important California weed, and cultivated radish (the same species), an important California crop (Klinger et al. 1991). (It is not unusual for a crop to be closely related to a weed of the same species.)

In 1988 and 1989, we grew the crop as if we were multiplying commercial seed and surrounded it with stands of

\section{GLOSSARY}

Allele: Type of a gene. For example, the common gene involved in human blood typing involves three alleles: A, B and $\mathrm{O}$.

Allozyme: Allelic protein products of a single gene that can be visualized with starch gel electrophoresis and subsequent appropriate staining.

Cultivated and wild types: Cultivated crops may have an array of wild relatives. (See box below.)

Fitness: Reproductive success as determined by survivorship and fecundity.

Gene flow: Migration of genes among populations. In humans, this involves migration of individuals. In plants, it may also involve pollen or seed movement.

Hybridization: Crossing among individuals from genetically distinct lineages. (See box below.)

Outcrossing: Reproduction that involves crossing between two different individuals. In humans and many animals, all reproduction is due to outcrossing. In some plants and animals, self-fertilization (selfing) is also an option, as well as reproduction without fertilization (asexuality).

Phenotype: Trait or traits expressed by an organism.

Taxon (taxa): Taxonomic unit, such as species or subspecies.

\section{A CLOSER LOOK}

\section{At hybridization ...}

Hybridization refers to crosses between individuals of different but related species. When this is the case, there are reproductive, isolating barriers that either reduce the chances of mating, such as differences in flowering, or reduce the chances of progeny passing on their genes, such as hybrid sterility (for example, the mule is a hybrid between the horse and the donkey, two separate species). Reproductive isolating barriers may be minimal or absent in the cases of hybridization between subspecies, between different varieties of the same crop, or between cultivated crops and crops that have gone wild (feral). Plant breeders often intentionally make artificial crosses to transfer beneficial traits. Spontaneous, natural hybridization is relatively rare in animal species, but is more common for plants.

\section{... and cultivated versus wild type}

Plants that are intentionally cultivated as crops may have an array of wild relatives. Some wild relatives may be the progenitors of the crop or other taxa whose ancestors were not cultivated. Also, some crops establish free-living (feral) populations. These populations may take off on their own evolutionary trajectory to become weeds of varying importance. In some cases, a single gene difference, such as bolting in beets, can change a crop into a weed. Finally, for many crops there are wild, weedy relatives that are descended from hybrids between cultivated plants and wild individuals without cultivated ancestors.

$$
\text { - N.C. Ellstrand }
$$


Right, sorghum is an important global food-grain crop, while its wild relative, far right, johnsongrass (Sorghum halepense), is one of U.S. agriculture's most troublesome weeds. Sorghum and johnsongrass have different numbers of chromosomes, but UC scientists found that they spontaneously hybridized when grown within 330 feet of each other.

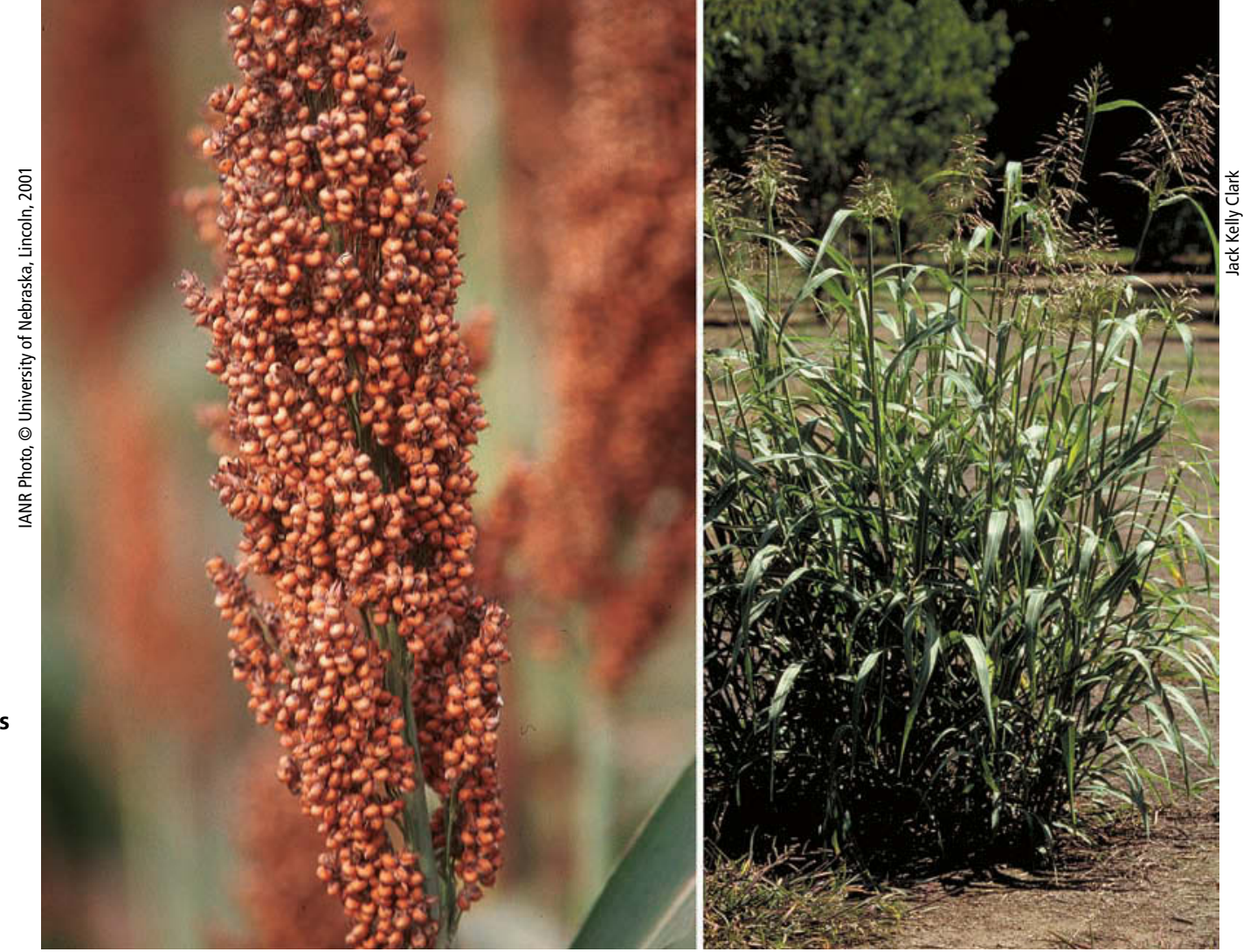

weeds at varying distances. When the plants flowered, pollinators did their job. We harvested seeds from the weeds for progeny testing. We exploited an allozyme allele that was present in the crop and absent in the weed to detect hybrids in the progeny of the weed. The experiment was repeated at the UC South Coast Research and Extension Center and at the UC Riverside Moreno Valley Field Station. At both locations, we found that many of the weed seeds analyzed at the shortest distance of 3.3 feet ( 1 meter) were sired by the crop (40\% hybridization), and that a low level (about $2 \%$ ) of hybridization was detected at the greatest distance of 0.62 mile (1 kilometer). It was clear, at least in this system, that crop alleles could enter natural populations.

But could they persist? The general view at that time was that hybrids of crops and weeds would be handicapped by crop characteristics that are agronomically favorable, but a detriment in the wild. The expectation was that crop-wild hybrids should have inferior fitness in the wild, compared to their wild parents.

We tested that view by comparing the fitness of the hybrids created in our first experiment with their nonhybrid siblings (Klinger and Ellstrand 1994). We grew them side by side under field conditions. The hybrids exhibited the huge, swollen root characteristic of the crop, but the pure wild plants did not. The two groups did not differ significantly in germination, survival or ability for their pollen to sire seed. However, the crop-wild hybrids set about $15 \%$ more seed than the wild plants. In this system, hybrid vigor would accelerate the spread of crop alleles in a natural population.

\section{Exception to the rule?}

When I presented these results at seminars, scientists questioned the generality of the results. "Isn't radish probably an exception?" they asked. "After all, radish is outcrossing and insect-pollinated. Its wild relative is the same species. What about a more important crop? What about a more important weed?"

We decided to address these criticisms with a different combination of crop and wild relative. Sorghum (Sorghum bicolor) is one of the world's most important crops, and johnsongrass (S. halepense) is one of the world's worst weeds. The two are distinct species, even differing in chromosome number, and sorghum is largely self- and wind-pollinated. The sorghum system was about as different from radish as you could get.

We conducted experiments with sorghum that paralleled those conducted with radish. We found that sorghum and johnsongrass spontaneously hybridized, although at rates lower than the radish system, and we detected crop alleles in seed set by wild plants growing 330 feet (100 meters) from the crop (Arriola and Ellstrand 1996). The fitness of the hybrids was not significantly different from their wild siblings (Arriola and Ellstrand 1997). The results from our sorghum-johnsongrass experiments were qualitatively the same as those from our cultivated radish-wild radish experiments.

Other labs have conducted similar experiments on crops such as sunflower (Helianthus annuus), rice (Oryza sativa), canola (Brassica napus) and pearl millet (Pennisetum glaucum) (Ellstrand 2003b; den Nijs et al. 2004). Almost all such studies obtained qualitatively similar results to those obtained by my research group. There are a few exceptions; for example, experiments have shown that potato (Solanum tuberosum) does not naturally mate with the wild species $S$. dulcamara and S. nigrum under field conditions (McPartlan and Dale 1994).

Additionally, descriptive studies conducted in my lab and others have often found crop-specific alleles in wild relatives when the two grow in proximity. In California, alleles from sugarbeets are found in populations of the wild beet Beta macrocarpa in the sugarbeet production region of the Imperial Valley, where the latter is a weed in and near sugar- continued on page 121 